\title{
A wire-loop technique for implantation of an iliac branched device in a patient with previous surgery for a ruptured abdominal aortic aneurysm
}

\author{
Umberto Marcello Bracale, Donatella Narese, Gaetano Vitale, Franco Maglione, Pietro Orlando Conti, \\ Guido Bajardi
}

\begin{abstract}
We described a modified technique for implanting a bridging stent-graft into an iliac branched device. A 79-year-old male who had received aortobiiliac synthetic graft surgery for a ruptured abdominal aortic aneurysm six months earlier was admitted to our unit for treatment of a left common iliac aneurysm involving the origin of the hypogastric artery. A standard technique was unsuccessful at implanting the bridging stent-graft, and therefore a wire-loop guidewire over the graft bifurcation was used to stabilize the contralateral sheaths and to complete the implantation.
\end{abstract}

From the Vascular and Endovascular Surgery Unit (U.M.B. $\bowtie$ umbertomarcello.bracale@unipa.it, D.N., G.V., P.O.C., G.B.) University of Palermo, Palermo, Italy; the Department of Diagnostic Imaging (F.M.), Section of General and Emergency Radiology, Cardarelli Hospital of Naples, Naples, Italy.

Received 8 February 2012; revision requested 1 April 2012; revision received 9 April 2012; accepted 18 April 2012.

Published online 24 September 2012

DOI 10.4261/1305-3825.DIR.5727-12.1 ntentional occlusion of one or both hypogastric arteries (HAs) during aortoiliac aneurysm repair is associated with several potential complications, including pelvic, colonic, and bowel ischaemia, hip and buttock claudication, and paraplegia (1). The risk of developing these complications increases after open aortic repair due to insufficient collateral flow to the inferior mesenteric artery (IMA) (2).

An iliac branched device (IBD) has been recently introduced as a new completely endovascular method for treating extensive aortoiliac or iliac aneurysms while concomitantly preserving HA flow. The IBD technology has evolved over the past decade and has demonstrated a low complication rate both during and after the procedure $(3,4)$.

In this case report, we described a modified technique for inserting a bridging stent-graft during an IBD placement for an iliac artery aneurysm in a patient who had previously received an aortobiiliac surgical graft for a ruptured aneurysm.

\section{Case report}

A 79-year-old male with a history of hypertension was referred to our department under the suspicion of a ruptured abdominal aortic aneurysm. Urgent computed tomography (CT) angiography showed a ruptured Type D aortoiliac aneurysm. The patient underwent open repair with an $18 \times 9 \mathrm{~mm}$ aortobiiliac Dacron graft implantation and surgical ligation of the IMA. The right graft limb was anastomosed on the right external iliac artery with concomitant ligation of the common iliac artery and HA. On the left side, the graft limb was anastomosed at the origin of the common iliac artery in order to preserve direct blood flow into the HA. The patient remained hemodynamically stable in the intensive care unit for three days and was discharged in good condition on the 12th postoperative day. At his six month follow-up examination, the patient was doing well, but a new CT angiography showed that the left iliac aneurysm had increased to its maximal diameter (from 34 to 42 $\mathrm{mm}$; Fig.1). We planned to treat the aneurysm by endovascular means with an IBD to maintain patient's last HA patent. The device used was Cook Zenith ZBIS (William Cook Europe, Bjaeverskov, Denmark) measuring $12 \times 61 \times 41 \mathrm{~mm}$. Under local anaesthesia, surgical exposure of both common femoral arteries was carried out. The 20 F IBD was introduced from the left access and the indwelling wire was snared from the contralateral access to achieve a through-and-through cross-over connection. We then attempted to advance a $55 \mathrm{~cm} 7 \mathrm{~F}$ Flexor sheath (William Cook Europe) through a $40 \mathrm{~cm} 10 \mathrm{~F}$ Flexor sheath (William Cook Europe), as previously described by Tielliu et al. (5), into the iliac side branch of the IBD from the right access, but this maneuver failed because of the angle at the level of the anastomotic site (Fig. 2a). Based on this complication, we decided to catheterize the HA with a $4 \mathrm{~F}$ Cobra glidecath (Terumo 

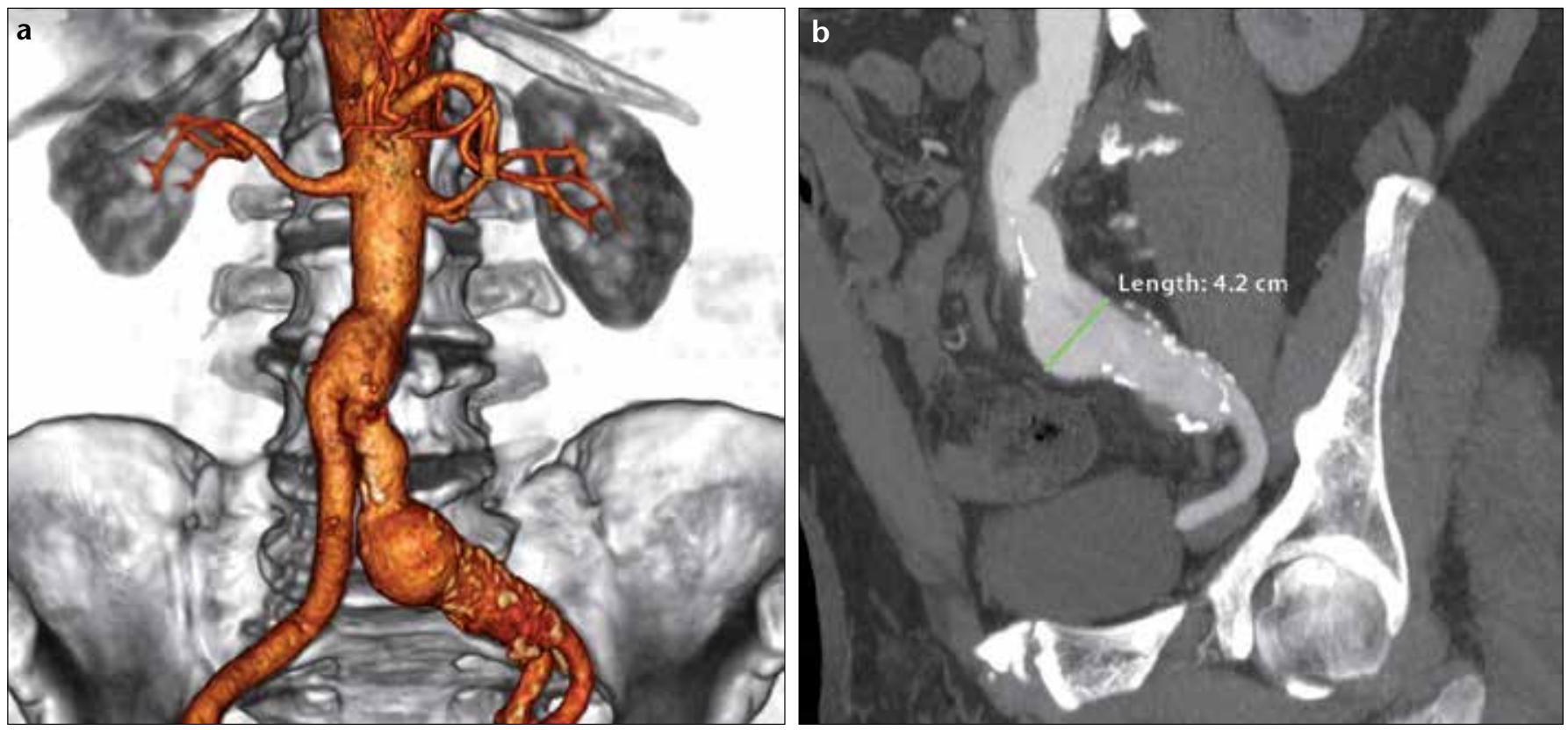

Figure 1. a, b. Preoperative CT angiography with a volume rendering three-dimensional image (a) and curved multiplanar reconstruction image (b), show the bifurcated graft and left common iliac aneurysm.
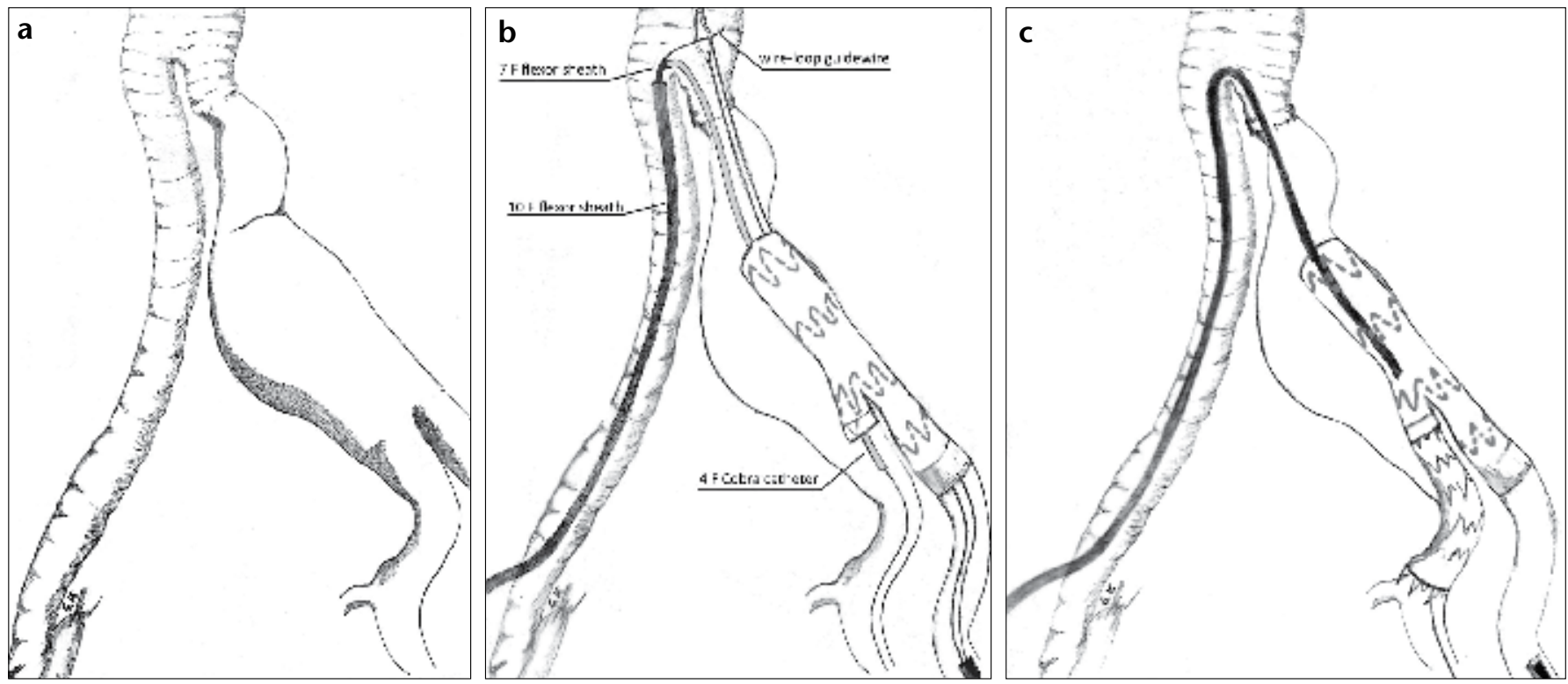

Figure 2. a-c. Illustration of the wire-loop technique in a patient with a previous bifurcated graft and left common iliac aneurysm (a) A soft wire is inserted through the $10 \mathrm{~F}$ Flexor sheath parallel to the $7 \mathrm{~F}$ Flexor sheath and snared from the left access, which makes a wire-loop over the graft bifurcation (b) The $10 \mathrm{~F}$ sheath is advanced into the iliac branched device with a gentle traction on the wire-loop, and a bridging stentgraft is guided over a stiff wire and deployed to fill the gap between the side branch of the iliac branched device and the hypogastric artery (c).

Medical Corporation, Somerset, New Jersey, USA) and a soft wire, and then release the entire IBD. A new soft wire was inserted through the $10 \mathrm{~F}$ Flexor sheath parallel to the $7 \mathrm{~F}$ Flexor sheath and snared from the left access making a wire-loop over the graft bifurcation (Fig. 2b and 2c). Using this approach, we were able to advance the $10 \mathrm{~F}$ sheath into the IBD with gentle traction on the wire loop. The Cobra catheter was advanced in a side branch of the HA and the soft wire was exchanged for an extra-stiff Supracore wire (Abbott Vascular, Abbott Park, Illinois, USA). A $9 \times 40 \mathrm{~mm}$ Fluency stentgraft (Bard Peripheral Vascular Inc., Murray Hill, New Jersey, USA) was guided over the stiff wire and deployed to fill the gap between the side branch of the IBD and the HA. Therefore, the wire-loop was removed and an iliac Cook Zenith stentgraft extension (TFLE 12-56-ZT, William Cook Europe) was employed to seal the proximal section of the IBD with the surgical graft limb. After ballooning, a completion angiogram demonstrated complete aneurysm exclusion and patency of the stent grafts (Fig. 3a).

There were no postoperative complications, and the patient was discharged four days after this uncomplicated 

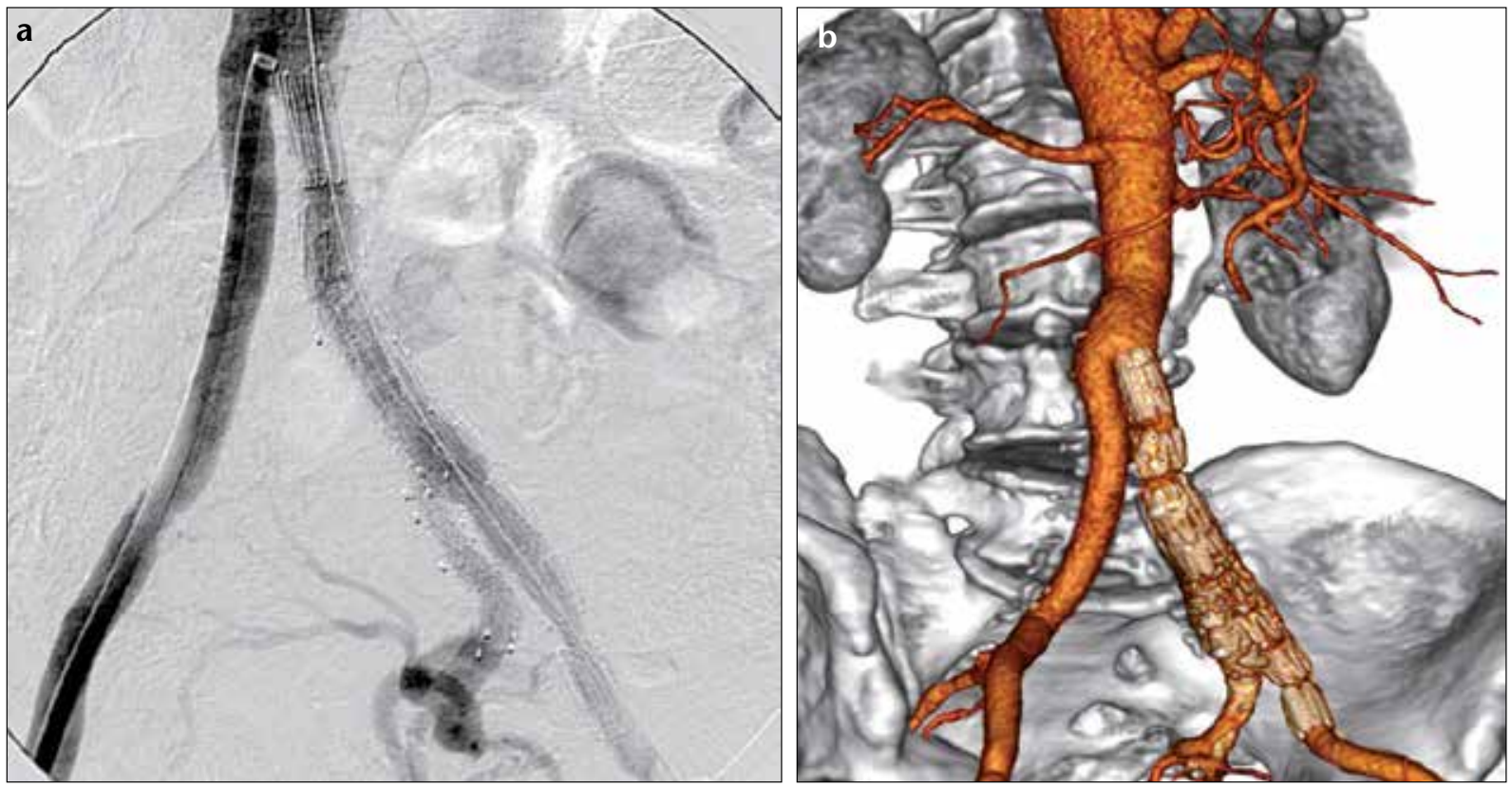

Figure 3. a, b. A completion angiography showing the patent hypogastric artery and exclusion of the aneurysm (a). CT angiography with volume rendering three-dimensional image 12 months after the intervention (b) demonstrates a sufficient stent-graft position and adequate hypogastric artery perfusion.

procedure. The follow-up CT angiography after one year showed no endoleak as well as adequate lower limb perfusion (Fig. 3b). At the time of the patient's last CT angiography, the patient was doing clinically well with no buttock or thigh claudication.

\section{Discussion}

When an aortoiliac aneurysm involves the iliac bifurcation (Type $D$ ), the use of a bifurcated prosthesis is required and standard surgical techniques, especially during emergency surgery, can include over sewing or the exclusion of the origins of the HAs. This is a relatively benign procedure, but it can sometimes lead to numerous complications, including buttock necrosis, lower extremity neurologic deficits, ischemic colitis, impotence, and buttock claudication. Several studies have reported a wide range of incidence rates of these complications, ranging from $28 \%$ to $55 \%$ in cases of bilateral HA occlusion $(6,7)$. Therefore, most clinicians aim to preserve blood flow in at least one of the HAs.

Some endovascular techniques have been described in recent studies that maintain antegrade flow in the HA. IBD is probably the best completely endovascular technique for preserving pelvic perfusion with low complication rates and durable aneurysm exclusion (4). However, this technology has some relative anatomical contraindications, such as iliac tortuosity or the presence of a bifurcated surgical graft. Vourliotakis et al. (8) described a similar case in which an axillary surgical approach was necessary to create a "body floss" support for a safe implantation of the bridging stent-graft in the IBD. Use of an axillary access can likely expand the application of the IBD in patients with tortuous anatomy, but placement of a 10-12 F sheath in this artery has its own risks, including vessel thrombosis or dissection as well as local wound complications. Moreover, most device components are not furnished with available lengths that would accommodate axillary access. In the case report described here, we used a wire-loop technique to overcome the friction at the anastomotic site of the surgical graft limb, which thereby avoided an extra access in the upper limb. To establish a stable wire-loop, we used a sheath large enough (10 Fr) to accommodate both the 0.035 inch hydrophilic soft wire and the parallel catheter system previously positioned in the target vessel.

In conclusion, IBD permits the endovascular treatment of complex iliac aneurysms while preserving HA flow. Importantly, technical tips and tricks, such as the wire-loop described in this report, can expand the applicability of this technology.

\section{Conflict of interest disclosure}

The authors declared no conflicts of interest.

\section{References}

1. Razavi MK, DeGroot M, Olcott C 3rd, et al. Internal iliac artery embolization in the stent-graft treatment of aortoiliac aneurysms: analysis of outcomes and complications. J Vasc Interv Radiol 2000; 11:561-566.

2. Paty PK, Shah DM, Chang BB, Darling RC 3rd, Leather RP. Pelvic ischemia following aortoiliac reconstruction. Ann Vasc Surg 1994; 8:204-206.

3. Tielliu IF, Bos WT, Zeebregts CJ, Prins TR, Van den Dungen JJ, Verhoeven EL. The role of branched endografts in preserving internal iliac arteries. J Cardiovasc Surg 2009; 50:213-218.

4. Parlani G, Verzini F, De Rango P, et al. Long-term results of iliac aneurysm repair with iliac branched endograft: a 5-year experience on 100 consecutive cases. Eur J Vasc Endovasc Surg 2012; 43:287-292.

5. Tielliu IF, Zeebregts CJ, van den Dungen JJ, Verhoeven EL. A modified technique for iliac artery branched endografting using a "tromboned" sheath. J Vasc Surg 2008; 48:1605-1608.

6. Mehta M, Veith FJ, Darling RC, et al. Effects of bilateral hypogastric artery interruption during endovascular and open aortoiliac aneurysm repair. J Vasc Surg 2004; 40:698-702.

7. Lin PH, Chen AY, Vij A. Hypogastric artery preservation during endovascular aortic aneurysm repair: is it important? Semin Vasc Surg 2009; 22:193-200.

8. Vourliotakis G, Bracale UM, Sondakh A, Tielliu IF, Prins T, Verhoeven EL. Iliac branched device implantation in tortuous iliac anatomy after previous open ruptured aortic aneurysm repair. J Cardiovasc Surg (Torino) 2012; 53:527-530. 